\title{
Serous retinal detachment in preeclampsia and malignant hypertension
}

\author{
Christopher Seungkyu Lee $\mathbb{D}^{1,2} \cdot$ Eun Young Choi ${ }^{1} \cdot$ Minsub Lee ${ }^{3} \cdot$ Heesuk Kim $^{2} \cdot$ Hyewon Chung ${ }^{3}$
}

Received: 13 November 2018 / Revised: 19 February 2019 / Accepted: 12 March 2019 / Published online: 14 May 2019

(c) The Royal College of Ophthalmologists 2019

\begin{abstract}
Objectives To compare and evaluate the characteristics of hypertensive choroidopathy with serous retinal detachment in preeclampsia and malignant hypertension (HTN) and explore choroidal ischemia as a pathogenesis using multimodal imaging.

Methods A retrospective multicenter case series. Medical charts were reviewed. Clinical characteristics and multimodal imaging, including optical coherence tomography (OCT) and OCT angiography (OCTA), were evaluated.

Results Fifty-three eyes of 29 preeclampsia patients and 45 eyes of 24 HTN patients were included. There were no differences in age, follow-up duration, baseline visual acuity, central macular thickness (CMT), or subfoveal choroidal thickness (CT) between the two groups. Blood pressure parameters, including systolic blood pressure, diastolic blood pressure, and pulse rate, were significantly higher in the HTN group. After serous retinal detachment resolved, both CMT ( $p$ $<0.001)$ and CT $(p=0.003)$ decreased more in the preeclampsia group. Hypertensive retinopathy features, including hemorrhage, exudates, cotton-wool spots, and optic disc edema, were predominantly found in the HTN group $(p=0.001)$. Final visual acuity was better in the preeclampsia group than in the HTN group $(p=0.048)$. Poor visual prognostic factors included the presence of retinopathy features $(p=0.005)$ and retinal detachment in the macula $(p=0.017)$.

Conclusion Choroidal circulation may be affected earlier than retinal circulation by elevated blood pressure, presumably because of anatomical differences and autoregulatory mechanisms in the retinal vasculature. Serous retinal detachment with hypertensive choroidopathy presented with choroidal thickening that decreased after resolution, but the residual flow defects observed in the choriocapillaris on OCTA confirmed the long-hypothesized notion that ischemia is a mechanism underlying hypertensive choroidopathy.
\end{abstract}

The study was presented in part at: The 18th European Vitreoretinal Society, August 30-September 2, 2018, Prague, Czech Republic.

\section{Hyewon Chung \\ hchung@kuh.ac.kr}

1 Department of Ophthalmology, The Institute of Vision Research, Gangnam Severance Hospital, Yonsei University College of Medicine, 211, Eonjuro, Gangnam-gu, Seoul, Republic of Korea

2 Department of Ophthalmology, The Institute of Vision Research, Severance Hospital, Yonsei University College of Medicine, 50-1, Yonseiro, Seodaemun-gu, Seoul, Republic of Korea

3 Department of Ophthalmology, Konkuk University School of Medicine, Konkuk University Medical Center, 120-1 Neungdongro, Gwangjin-gu, Seoul, Republic of Korea

\section{Introduction}

Preeclampsia is an obstetric complication characterized by hypertension, edema, and proteinuria that has an estimated incidence of 3-5\% of pregnancies [1]. Eclampsia is defined as preeclampsia with seizures and occurs late in pregnancy. Hypertensive choroidopathy clinically presents with serous retinal detachments (SRDs) and yellowish retinal pigment epithelium (RPE) lesions and can complicate preeclampsia or eclampsia. Serous retinal detachments are typically bilateral and bullous and cause marked visual loss, but these changes usually resolve postpartum with the recovery of normal vision. Severe cases can, however, result in geographic chorioretinal atrophy, which occurs in up to $8.5 \%$ of preeclampsia patients $[2,3]$. Based on limited histopathological and clinical studies, the mechanism underlying choroidopathy is thought to be ischemia [4-9]. Choriocapillaris nonperfusion and choroidal thickening with 
secondary RPE changes are consistently found in affected patients. The pathophysiology of choroidal thickening in a setting of choroidal ischemia is not fully understood. Clues may be found in the various choroid-thickening entities that are collectively referred to by some authors as "pachychoroid" diseases [10-14]. We previously showed that choroidal thickness (CT) was increased and large choroidal vessels dilated on enhanced-depth imaging optical coherence tomography (EDI-OCT) [14]; these presentations are similar to those observed in other pachychoroid spectrum diseases, such as pachychoroid neovasculopathy and central serous chorioretinopathy. Increased CT has also been demonstrated in preeclampsia patients in the absence of evident choroidopathy features [15].

Few studies have explored hypertensive choroidopathy with SRD in preeclampsia or eclampsia, and most have been anecdotal case reports or series. To evaluate the clinical features of serous retinal detachments in preeclampsia/eclampsia choroidopathy, we sought to compare their clinical findings to serous retinal detachments observed in malignant hypertension, another wellrecognized cause of hypertensive choroidopathy. The aim of the present study was to use multimodal imaging technologies to evaluate and compare the clinical course and features associated with SRDs in preeclampsia/ eclampsia and malignant hypertension. Special emphasis will be placed on analyzing choroidal imaging to identify evidence for choroidal ischemia in these entities, as has been historically hypothesized.

\section{Methods}

\section{Subjects}

We retrospectively reviewed the medical records of patients who were evaluated and treated for SRD among patients who were diagnosed with severe preeclampsia or uncontrolled systemic hypertension in the Department of Ophthalmology at Yonsei University Severance Medical Center between November 2009 and May 2017 and in the Department of Ophthalmology at Konkuk University Medical Center between February 2013 and February 2018. Institutional Review Board (IRB) approval was obtained at each site, and the study complied with the guidelines of the Declaration of Helsinki.

We reaffirmed the diagnosis of severe preeclampsia according to previously described criteria [1, 16]. Women with a clinical diagnosis of pregestational or gestational hypertension were excluded. The malignant hypertension group included patients with high blood pressure (systolic blood pressure $(\mathrm{SBP}) \geq 140 \mathrm{mmHg}$ or diastolic pressure $(\mathrm{DBP}) \geq 90 \mathrm{mmHg}$ ) and hypertensive retinopathy features, including retinal hemorrhage, exudates, cotton-wool spots, diffuse retinal edema, and optic disc edema. [17]

We reviewed the results of ophthalmologic examinations performed in all subjects, including fundus examination by retinal specialists, color fundus photography, and EDI-OCT (Spectralis OCT; Heidelberg Engineering Inc., Heidelberg, Germany). Some patients underwent fluorescein angiography, indocyanine green angiography, or OCT angiography (OCTA) (Angio-Plex, CIRRUS HD-OCT models 5000, Carl Zeiss Meditec, Inc., Dublin, CA, USA; Optovue RTVue XR Avanti, Optovue, Inc., Freemont, CA, USA; Spectralis OCT2, Heidelberg Engineering Inc., Heidelberg, Germany). We included only those patients who were confirmed to have subretinal fluid on OCT images in one or both eyes.

\section{Main outcome}

Basic parameters, such as age, sex, coexisting diseases, and follow-up duration, were collected. Obstetric information, such as parity, last menstrual period, delivery date, mode of delivery, and associated complications, was noted. We calculated body mass index (BMI) in the patients using measurements of height and weight. SBP, DBP, and pulse rate (PR) data were collected. We recorded baseline laboratory results, including the following: complete blood cell counts with differentials, platelet counts, routine blood biochemistry tests, and urinalysis.

Horizontal volume scans and EDI horizontal foveal scans obtained on spectral domain OCT were used to obtain measurements of central macular thickness (CMT) and subfoveal CT, respectively. CMT was automatically measured after segmentation of the inner limiting membrane and Bruch's membrane layer using Heidelberg software (version 6.7; Heidelberg Engineering). The choroid was defined as the region from the outer portion of the hyperreflective line corresponding to the RPE to the inner surface of the sclera [18].

\section{Statistical analysis}

All data are presented as the mean \pm standard deviation. Kolmogorov-Smirnov tests were used to analyze the distributions of samples. To compare baseline demographic and clinical characteristics and ophthalmic measurements between the two groups, we used the Chi-square test for categorized parameters and independent $t$-tests for continuous variables. We used paired $t$-tests to analyze changes in CMT and CT between the initial and final time points within each group. To examine the relationships between baseline parameters and the changes in CMT and CT observed in each group, a Pearson correlation test was performed. All statistical analyses were performed using 
SPSS version 21.0 (SPSS Inc., Chicago, IL, USA). A $P$ value of $<0.05$ was considered statistically significant.

\section{Results}

In the current study, SRDs were observed in 53 eyes of 29 patients with preeclampsia and 45 eyes of 24 patients with malignant hypertension. Of the 29 patients with preeclampsia, 4 progressed to eclampsia with seizures. SRDs

Table 1 Demographic data and laboratory results in preeclampsia and malignant hypertension patients with serous retinal detachment

\begin{tabular}{|c|c|c|c|}
\hline & Preeclampsia & $\begin{array}{l}\text { Malignant } \\
\text { hypertension }\end{array}$ & $P$ value \\
\hline Patients, $N$ & 29 & 24 & \\
\hline Age (years) & $33.33 \pm 3.44$ & $37.29 \pm 9.09$ & $0.52^{*}$ \\
\hline $\operatorname{Sex}(\mathrm{F} / \mathrm{M})$ & $18 / 0$ & $13 / 11$ & $<0.001$ \\
\hline DM history (yes/no) & $4 / 29$ & $4 / 21$ & $0.44^{\dagger}$ \\
\hline $\operatorname{BMI}\left(\mathrm{kg} / \mathrm{m}^{2}\right)$ & $25.44 \pm 4.46$ & $25.70 \pm 5.72$ & $0.85^{*}$ \\
\hline $\begin{array}{l}\text { Systolic blood pressure } \\
(\mathrm{mm} \mathrm{Hg})\end{array}$ & $155.63 \pm 22.01$ & $202.63 \pm 31.68$ & $<0.001$ \\
\hline $\begin{array}{l}\text { Diastolic blood pressure } \\
(\mathrm{mm} \mathrm{Hg})\end{array}$ & $97.90 \pm 14.11$ & $123.50 \pm 22.91$ & $<0.001$ \\
\hline Pulse rate $(\mathrm{bpm})$ & $84.90 \pm 20.03$ & $96.82 \pm 13.92$ & 0.017 \\
\hline \multicolumn{4}{|c|}{ Etiology of hypertension, $N(\%)$} \\
\hline Essential & - & $9(37.5)$ & \\
\hline Renal artery stenosis & - & $1(4.2)$ & \\
\hline $\begin{array}{l}\text { Renal parenchymal } \\
\text { disease }\end{array}$ & - & $5(20.8)$ & \\
\hline $\begin{array}{l}\text { Chronic kidney } \\
\text { disease }(\mathrm{CKD})\end{array}$ & - & $9(37.5)$ & \\
\hline \multicolumn{4}{|c|}{ Pregnancy complication classification, $N(\%)$} \\
\hline HELLP incomplete & $8(27.6)$ & & \\
\hline HELLP complete & $4(13.8)$ & & \\
\hline $\begin{array}{c}\text { Eclamptic } \\
\text { seizure event }\end{array}$ & $4(13.8)$ & & \\
\hline \multicolumn{4}{|l|}{ Clinical laboratory results } \\
\hline $\begin{array}{l}\text { Proteinuria (dipstick } \\
\text { positive) }\end{array}$ & $2.54 \pm 0.83$ & $1.77 \pm 1.30$ & 0.020 \\
\hline BUN (mg/dL) & $15.50 \pm 6.64$ & $38.57 \pm 10.91$ & 0.001 \\
\hline Creatinine (mg/dL) & $0.87 \pm 0.41$ & $5.23 \pm 8.99$ & 0.026 \\
\hline Platelets $(/ \mathrm{mL})$ & $153.91 \pm 66.93$ & $224.50 \pm 89.64$ & 0.003 \\
\hline $\begin{array}{l}\text { Aspartate } \\
\text { transaminase (U/L) }\end{array}$ & $73.97 \pm 17.03$ & $24.79 \pm 14.53$ & $0.23^{*}$ \\
\hline $\begin{array}{l}\text { Alanine amino } \\
\text { transferase (U/L) }\end{array}$ & $46.12 \pm 13.76$ & $23.66 \pm 16.74$ & $0.29^{*}$ \\
\hline
\end{tabular}

Values are presented as the mean \pm standard deviation. Independent $t$-tests* and Chi-square tests $\dagger$ were used to compare the groups. $P$ values in bold indicate significant differences.

$D M$ diabetes mellitus, BMI body mass index, HELLP hemolysis $(\mathrm{H})$, elevated liver enzymes (EL) and low platelet count (LP) were diagnosed during pregnancy in 16 eyes of $9(30.2 \%)$ preeclampsia patients and postpartum in 37 eyes of 20 $(69.8 \%)$ preeclampsia patients. The baseline demographic and clinical characteristics of the patients are presented in Tables 1 and 2. There were no significant differences in age, history of diabetes, or BMI between the two groups.

Table 2 Clinical characteristics and course results in preeclampsia and malignant hypertension patients with serous retinal detachment

\begin{tabular}{|c|c|c|c|}
\hline & Preeclampsia & $\begin{array}{l}\text { Malignant } \\
\text { hypertension }\end{array}$ & $P$ value \\
\hline Eyes with SRD, $N$ & 53 & 45 & \\
\hline Laterality (Rt/ Lt) & $28 / 25$ & $22 / 23$ & $0.73^{\dagger}$ \\
\hline \multicolumn{4}{|l|}{$\begin{array}{l}\text { Onset of SRD, } \\
N(\%)\end{array}$} \\
\hline $\begin{array}{c}\text { During } \\
\text { pregnancy }\end{array}$ & $16(30.2)$ & - & \\
\hline Postpartum & $37(69.8)$ & - & \\
\hline $\begin{array}{l}\text { Follow-up duration } \\
\text { (months) }\end{array}$ & $1.83 \pm 2.16$ & $2.15 \pm 2.65$ & $0.57^{*}$ \\
\hline \multicolumn{4}{|l|}{ CDVA (logMAR) } \\
\hline Baseline & $0.24 \pm 0.37$ & $0.28 \pm 0.44$ & $0.68^{*}$ \\
\hline Final & $0.11 \pm 0.16$ & $0.22 \pm 0.49$ & $0.048^{*}$ \\
\hline $\begin{array}{l}\text { Spherical } \\
\text { equivalent (diopter) }\end{array}$ & $-0.44 \pm 1.40$ & $-0.20 \pm 1.21$ & $0.74^{*}$ \\
\hline IOP $(\mathrm{mm} \mathrm{Hg})$ & $13.27 \pm 3.22$ & $14.73 \pm 4.18$ & $0.06^{*}$ \\
\hline $\begin{array}{l}\text { Location of SRD, } \\
N(\%)\end{array}$ & & & $0.73^{\dagger}$ \\
\hline Peripapillary & $47(88.7)$ & 39 (86.7) & \\
\hline Macular & $43(81.1)$ & $32(71.1)$ & \\
\hline Both & 37 (69.8) & $27(60.0)$ & \\
\hline
\end{tabular}

Presence of

$<0.001^{\dagger}$ retinopathy, $N(\%)$

$\begin{array}{lll}\text { Yes } & 8(15.1) & 44(97.8) \\ \text { No } & 45(84.9) & 1(2.2)\end{array}$

Central macular thickness $(\mu \mathrm{m})$

$\begin{array}{clll}\text { Baseline } & 464.69 \pm 347.75 & 355.42 \pm 12.66 & 0.45^{*} \\ & (197-1310) & (193-654) & \\ \text { Final } & 218.86 \pm 32.20 & 265.75 \pm 26.17 & <0.001^{*} \\ & (156-290) & (197-336) & \\ \text { P value } & <0.001^{*} & <0.001^{+} & \end{array}$

Subfoveal choroidal thickness $(\mu \mathrm{m})$

\begin{tabular}{llll} 
Baseline & $338.10 \pm 10.66$ & $318.71 \pm 13.16$ & $0.35^{*}$ \\
& $(126-665)$ & $(96-552)$ & \\
Final & $252.52 \pm 13.16$ & $289.36 \pm 14.89$ & $0.06^{*}$ \\
& $(126-565)$ & $(92-468)$ & \\
value & $<0.001^{*}$ & $\mathbf{0 . 0 0 2}^{*}$ & \\
\hline
\end{tabular}

Values are presented as the mean \pm standard deviation (range). Independent $t$-tests* and Chi-square tests ${ }^{\dagger}$ were used to compare the groups, and a paired $t$-test ${ }^{\ddagger}$ was used to analyze periodic changes within each group. $P$ values in bold indicate statistically significant differences.

$S R D$ serous retinal detachment, $C D V A$ corrected distance visual acuity, IOP intraocular pressure 

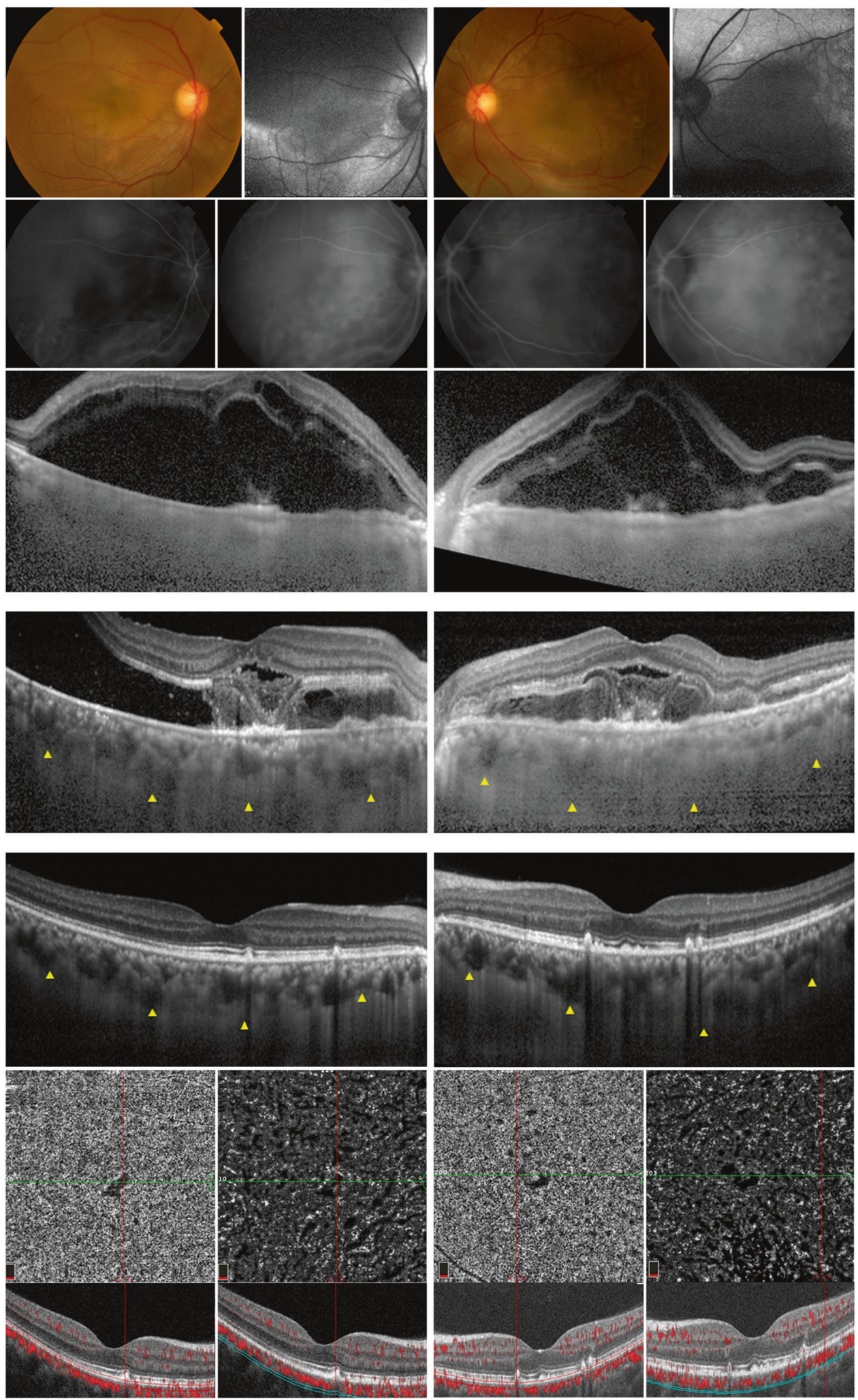

However, blood pressure profiles, including SBP, DBP, and $\mathrm{PR}$, were significantly higher in the malignant hypertension group than in the preeclampsia group $(p<0.001, p<0.001$, and $p=0.017$, respectively). At the end of the follow-up period, there were no patients with uncontrolled blood pressure in either groups. Thrombocytopenia $(p=0.003)$ and proteinuria $(p=0.020)$ were more severe in the preeclampsia group, but blood urea nitrogen (BUN) and 
Fig. 1 A 34-year-old woman with preeclampsia. Corrected distance visual acuity (CDVA) was 20/30 in the right eye and 20/50 in the left eye on the day of delivery. Her blood pressure was $159 / 94 \mathrm{~mm} \mathrm{Hg}$ at initial presentation. (Top row) Serous retinal detachments and retinal edema were found in both eyes in color fundus photos (first and third images) and fundus autofluorescence images (second and fourth images). No retinal hemorrhage or cotton-wool spots were observed. (Second row) Indocyanine green angiography showed early choroidal filling delay (first and third images) and late choroidal hyperpermeability (second and fourth images). (Third row) Large amounts of subretinal and retinal fluid obscured the choroid in both eyes on enhanced-depth imaging optical coherence tomography (EDI-OCT). (Fourth row) 3 days after delivery, EDI-OCT showed that the amounts of subretinal and retinal fluid with fibrinoid exudation were decreased in both eyes. Yellow triangles indicate the margin of the choroidal layer. Choroidal thickness was $488 \mu \mathrm{m}$ in the right eye (left image) and $504 \mu \mathrm{m}$ in the left eye (right image). (Fifth row) 4 months later, her CDVA improved to $20 / 20$ in both eyes. Multiple outer retinal and retinal pigment epithelial (RPE) lesions remained following the resolution of subretinal and retinal fluid. Choroidal thickness decreased to $302 \mu \mathrm{m}$ in the right eye (left image) and $308 \mu \mathrm{m}$ in the left eye (right image). (Bottom row) OCT angiography (OCTA) revealed an absence of flow signal at the level of the choriocapillaris in both maculae. A corresponding OCT scan acquired at the level of the green line shown on the OCTA image confirmed that this flow void area at the level of the choriocapillaris was not caused by signal attenuation due to overlying hyperreflective lesions (red line). The extent of choriocapillaris involvement exceeded the detectable outer retinal and RPE structural changes. No significant abnormalities were found at the level of Haller's layer in either macula

creatinine levels were both higher in hypertension patients ( $p=0.001$ and $p=0.026$, respectively).

There were no differences in baseline corrected distance visual acuity, intraocular pressure, or spherical equivalent between the two groups. Visual acuity increased in both groups but did so more prominently in the preeclampsia group, with $\log$ MAR visual acuity increasing from $0.24 \pm$ 0.37 (Snellen equivalent of 20/35) to $0.11 \pm 0.16$ (Snellen equivalent of 20/26) in the preeclampsia group $(p=0.001)$ during a mean follow-up of $1.83 \pm 2.16$ months and from $0.28 \pm 0.44$ (Snellen equivalent of 20/38) to $0.22 \pm 0.49$ (Snellen equivalent of 20/33) in the malignant hypertension group $(p=0.016)$ during a mean follow-up of $2.15 \pm$ 2.65 months. Only four eyes $(7.5 \%)$ in the preeclampsia group showed a final visual acuity less than 20/40, but the ten eyes $(22.2 \%)$ with malignant hypertension had a final visual acuity of less than 20/40. Risk factors for a poor visual prognosis included the presence of retinopathy $(p=$ $0.005)$ and the macular location of retinal detachment $(p=$ $0.017)$.

There were no differences in baseline CMT and CT between the two groups. After SRD was resolved, CMT and CT decreased in both groups but did so more prominently in the preeclampsia group. CMT decreased by $52.9 \%$, from $464.69 \pm 347.75 \mu \mathrm{m}$ to $218.86 \pm 32.20 \mu \mathrm{m}(p<0.001)$, in the preeclampsia group and by $25.3 \%$, from $355.42 \pm 12.66$ $\mu \mathrm{m}$ to $265.75 \pm 26.17 \mu \mathrm{m}(p<0.001)$, in the malignant hypertension group. The difference in the degree of CMT change was significant between the groups $(245.82 \mu \mathrm{m}$ versus $107.14 \mu \mathrm{m} ; p<0.001)$. Subfoveal CT decreased by $25.4 \%$, from $338.10 \pm 10.66 \mu \mathrm{m}$ to $252.52 \pm 13.16 \mu \mathrm{m}$ ( $p<$ 0.001 ), in the preeclampsia group and by $9.1 \%$, from $318.71 \pm 13.16 \mu \mathrm{m}$ to $289.36 \pm 14.89 \mu \mathrm{m}(p=0.002)$, in the malignant hypertension group. The difference in the degree of CT change was significant between the groups (110.82 $\mu \mathrm{m}$ versus $34.04 \mu \mathrm{m} ; p=0.003$ ).

Hypertensive retinopathy features, such as retinal hemorrhage, exudates, cotton-wool spots, diffuse retinal edema, and optic disc edema, were presented in only 8 $(15.1 \%)$ preeclampsia eyes but in $44(97.8 \%)$ malignant hypertensive eyes $(p=0.001)$. The factors positively associated with the presence of hypertensive retinopathy features included SBP $(p<0.0001)$, DBP $(p<0.0001)$, PR $(p=0.021)$, and the degree of CMT change $(p=0.017)$. Representative cases of preeclampsia and malignant hypertension are shown in Figs. 1 and 2.

\section{Discussion}

To our knowledge, this is the first study to use multimodal retinal imaging to evaluate and compare clinical manifestations in SRDs in preeclampsia and malignant hypertension patients. This study demonstrated that blood pressure profiles, including SBP, DBP, and PR, were significantly lower in preeclampsia patients than in malignant hypertension patients. The mean SBP was $155.63 \mathrm{~mm} \mathrm{Hg}$ in preeclampsia patients, in whom it rarely increased to over $180 \mathrm{~mm} \mathrm{Hg}$, whereas the mean SBP was $202.63 \mathrm{~mm} \mathrm{Hg}$ in malignant hypertensive patients. While hypertensive choroidopathy features with markedly thickened choroid and SRDs [9] were observed in both groups, hypertensive retinopathy features, such as hemorrhage, exudates, cotton-wool spots, diffuse retinal edema, and optic disc edema, were predominantly present in the malignant hypertension patients. Although preeclampsia-induced hypertension and malignant hypertension may not share a common pathophysiology, this finding may suggest that retinopathy is associated with even higher blood pressure levels than are found in choroidopathy. Preeclampsia patients could be viewed as having not achieved a "malignant" threshold level toward the development of retinopathy. The copresence of choroidopathy and retinopathy features in some preeclampsia patients may be a manifestation of preeclampsia choroidopathy superimposed on preexisting hypertension rather than the concurrent development of both [19]. The statistical association revealed in this study between the presence of hypertensive retinopathy features and increased blood pressure adds support to this hypothesis. There are several 
explanations for why the choroidal vasculature is affected earlier in hypertension. Anatomically, the choroidal arteries are not highly branched and run a relatively short course at a right angle to supply the choriocapillaris; thus, blood pressure is transmitted more directly to choriocapillaris [20-22]. Functionally, the choroidal vasculature has few autoregulatory properties and is controlled primarily by the sympathetic nervous system, while the
Fig. 2 A 38-year-old man with malignant hypertension. The corrected distance visual acuity was $20 / 20$ in both eyes, and his blood pressure was $180 / 123 \mathrm{~mm}$ $\mathrm{Hg}$ at the initial presentation. (Top row) Color fundus photos showed multiple retinal hemorrhages, cotton-wool spots and signs of hypertensive retinopathy (first and third images), and fluorescence angiography showed multiple late-phase leakage (second and fourth images). (Second row) Indocyanine green angiography showed early choroidal filling delay (first and third images) and middle- to late-phase multiple hyperfluorescent spots (second and fourth images). (Third row) On enhanced-depth imaging optical coherence tomography (EDI-OCT) performed through the macula at the initial examination, mild outer retinal edema was observed in the macula nasal to the fovea in the right eye (left image), and serous retinal detachment and outer retinal edema were present in the left eye (right image). Yellow triangles indicate the margin of the choroidal layer. Choroidal thickness was $372 \mu \mathrm{m}$ in the right eye and $349 \mu \mathrm{m}$ in the left eye. (Fourth row) Serous retinal detachment and outer retinal edema improved at 3 weeks, when his blood pressure returned to normal. Choroidal thickness decreased to $300 \mu \mathrm{m}$ in the right eye (left image) and $309 \mu \mathrm{m}$ in the left eye (right image). (Fifth row) One year later, no definite outer retinal or RPE structural changes were found in either eye on EDIOCT; however, (bottom row) multiple areas of decreased flow signal were observed at the level of the choriocapillaris in both maculae on OCT angiography
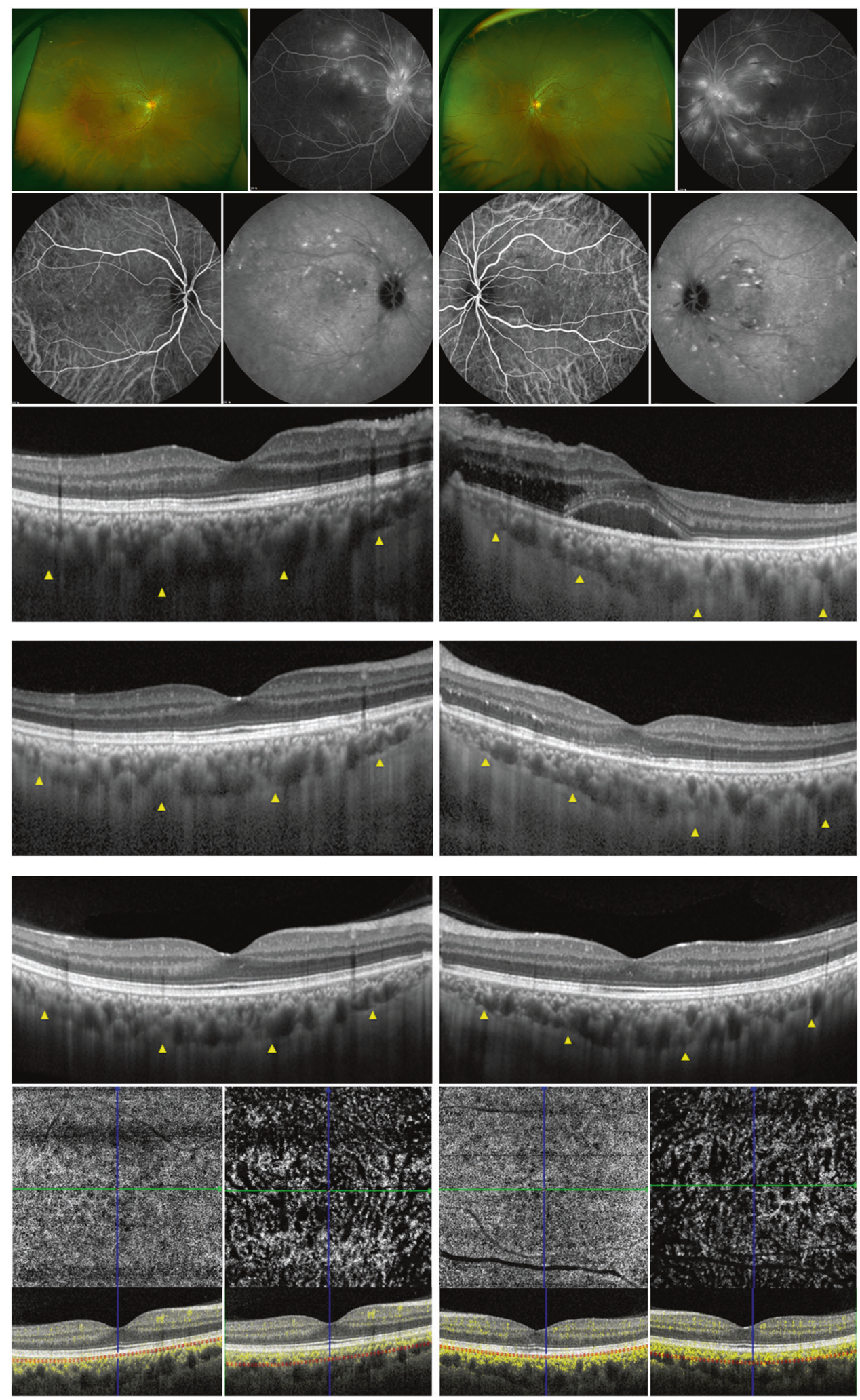
retinal circulation is governed by autoregulatory mechanisms. Hence, if elevated blood pressure overcomes the compensatory sympathetic response, it can damage the choroidal vasculature [23], whereas retinal vessels are able to tolerate much higher blood pressure while maintaining vascular tone because they possess autoregulatory mechanisms [22]. However, it must be noted that preeclampsia and malignant hypertension are marked by different pathophysiologies; thus, the differences in their ocular manifestations should not be expected to be solely dependent on their differences in blood pressure levels.

It is worth noting that the degree of SRD and increases in $\mathrm{CT}$ were more pronounced in preeclampsia patients than in malignant hypertension patients. The reason that preeclampsia patients are marked primarily by choroidopathy but not retinopathy may not be entirely due to their less malignant blood pressure levels. Abnormal placentation causes chronic placental ischemia and oxidative stress, inducing the release of substances into the maternal circulation; these include free radicals, oxidized lipids, and antiangiogenic factors, such as soluble Flt1, which are responsible for the generalized endothelial dysfunction seen in preeclampsia [24-27]. Generalized vasospasm is another characteristic of preeclampsia [26, 28, 29]. Endothelial dysfunction and vasospasm of the choroidal circulation could cause fibrinoid necrosis of choroidal vessels with occlusion of the choriocapillaris, leading to necrosis of the overlying RPE and the development of SRD in preeclampsia choroidopathy [22]. Retinal arteriolar constriction is also commonly observed in preeclampsia, presumably as a result of retinal artery vasospasm, as suggested by the increased central retinal artery blood flow velocity observed in affected patients [30]; however, it may be well tolerated by retinal autoregulation, as previously mentioned. The choroidal thickening observed in preeclampsia could be caused primarily by venous congestion, which can be inferred from kidney tests because the kidneys and eyes share striking similarities in terms of their structural, physiological, and pathogenic pathways [31]. In the glomeruli of preeclampsic women, resistance in afferent arterioles is substantially increased, but efferent resistance is not as changed [32]. We speculate that choroidal resistance at the level of the large venular side might also not be increased in preeclampsia.

Visual outcomes were favorable in preeclampsia patients and variable in malignant hypertensive patients. A poor visual prognosis was associated with the presence of retinopathy and a macular location of SRDs. After SRDs were resolved, CT decreased, but areas of flow signal attenuation were observed at the level of the choriocapillaris on OCTA and found to overlie dilated choroidal vessels in some patients (Figs. 1 and 2). In some areas with reduced inner choroid flow, this flow seemed to be responsible for inner choroidal thinning under acute conditions and manifested as areas of inner choroidal ischemia.

In conclusion, the results of the present study illustrate the differences between the clinical manifestations of patients with choroidopathy in preeclampsia and those with malignant hypertension. SRD and choroidal thickening were more pronounced in preeclampsia choroidopathy but resulted in more favorable visual outcomes when observed in the absence of retinopathy features, including hemorrhage, exudates, and cotton-wool spots. Choroidal thickening accompanied by the dilation of large choroidal vessels and hyperpermeability could be caused by choroidal ischemia related to endothelial dysfunction, vasospasm, and systemic hypertension, as demonstrated in these patients.

\section{Summary}

\section{What was known before}

- SRD develops in moderate hypertension in preeclampsia.

- The clinical features of preeclampsia/eclampsia choroidopathy in SRD have not been fully investigated.

\section{What this study adds}

- Elevated blood pressures affect the choroid earlier than the retina.

- Choroid thickness decreases after hypertensive choroidopathy resolves.

- Additional retinopathy in hypertensive choroidopathy carries a poorer visual prognosis.

Funding This research was supported by the National Research Foundation of Korea (NRF) (NRF-2017R1E1A1A01073964 and NRF-2016R1D1A1A02937349), which is funded by the Ministry of Science and ICT.

\section{Compliance with ethical standards}

Conflict of interest The authors declare that they have no conflict of interest.

Publisher's note: Springer Nature remains neutral with regard to jurisdictional claims in published maps and institutional affiliations.

\section{References}

1. Mol BWJ, Roberts CT, Thangaratinam S, Magee LA, de Groot CJM, Hofmeyr GJ. Pre-eclampsia. Lancet. 2016;387:999-1011. 
2. Sunness JS. The pregnant woman's eye. Surv Ophthalmol. 1988;32:219-38.

3. Saito Y, Tano Y. Retinal pigment epithelial lesions associated with choroidal ischemia in preeclampsia. Retina. 1998;18:103-8.

4. Hayreh SS. Acute occlusive disorders of the choroidal vasculature. Int Ophthalmol. 1983;6:139-48.

5. Song YS, Kinouchi R, Ishiko S, Fukui K, Yoshida A. Hypertensive choroidopathy with eclampsia viewed on spectral-domain optical coherence tomography. Graefes Arch Clin Exp Ophthalmol. 2013;251:2647-50.

6. Oliver M, Uchenik D. Bilateral exudative retinal detachment in eclampsia without hypertensive retinopathy. Am J Ophthalmol. 1980;90:792-6.

7. Gass DM, Pautler SE. Toxemia of pregnancy pigment epitheliopathy masquerading as a heredomacular dystrophy. Trans Am Ophthalmol Soc. 1985;83:114-30.

8. Querques L, Querques G, Loperfido F, Lattanzio R, Bandello F. Enhanced depth imaging optical coherence tomography findings associated with serous retinal detachment in preeclampsia. Arch Gynecol Obstet. 2014;289:457-9.

9. Hayreh SS, Servais GE, Virdi PS. Fundus lesions in malignant hypertension. VI. Hypertensive choroidopathy. Ophthalmology. 1986;93:1383-1400.

10. Warrow DJ, Hoang QV, Freund KB. Pachychoroid pigment epitheliopathy. Retina. 2013;33:1659-72.

11. Pang CE, Freund KB. Pachychoroid neovasculopathy. Retina. 2015;35:1-9.

12. Dansingani KK, Balaratnasingam C, Naysan J, Freund KB. En face imaging of pachychoroid spectrum disorders with sweptsource optical coherence tomography. Retina. 2016;36:499-516.

13. Gallego-Pinazo R, Dolz-Marco R, Gomez-Ulla F, Mrejen S, Freund KB. Pachychoroid diseases of the macula. Med Hypothesis Discov Innov Ophthalmol. 2014;3:111-5.

14. Chung H, Byeon SH, Freund KB. Focal choroidal excavation and its association with pachychoroid spectrum disoRDERS: a review of the literature and multimodal imaging findings. Retina. 2017;37:199-221.

15. Garg A, Wapner RJ, Ananth CV, Dale E, Tsang SH, Lee W, et al. Choroidal and retinal thickening in severe preeclampsia. Investig Ophthalmol Vis Sci. 2014;55:5723-9.

16. National High Blood Pressure Education Program Working Group Report on High Blood Pressure in Pregnancy. Am J Obstet Gynecol. 1990;163(5 Pt 1):1691-712. https://www.ncbi.nlm.nih. gov/pubmed/2104525.
17. Erden S, Bicakci E. Hypertensive retinopathy: incidence, risk factors, and comorbidities. Clin Exp Hypertens. 2012;34:397-401.

18. Spaide RF, Koizumi H, Pozzoni MC. Enhanced depth imaging spectral-domain optical coherence tomography. Am J Ophthalmol. 2008;146:496-500.

19. Saito Y, Omoto T, Kidoguchi K, Fujita T, Wada Y. The relationship between ophthalmoscopic changes and classification of toxemia in toxemia of pregnancy. Nippon Ganka Gakkai Zasshi. 1990;94:870-4.

20. Hayreh SS. Vascular pattern of the choriocapillaris. Exp Eye Res. 1974;19:101-4.

21. Hayreh SS. The choriocapillaris. Albrecht Von Graefes Arch Klin Exp Ophthalmol. 1974;192:165-79.

22. Tso MO, Jampol LM. Pathophysiology of hypertensive retinopathy. Ophthalmology. 1982;89:1132-45.

23. Weiter JJ, Schachar RA, Ernest JT. Control of intraocular blood flow. II. Effect sympathetic tone. Investig Ophthalmol Vis Sci. 1973;12:332-4.

24. Steegers EA, von Dadelszen P, Duvekot JJ, Pijnenborg R. Preeclampsia. Lancet. 2010;376:631-44.

25. Kobayashi $\mathrm{T}$, Tokunaga $\mathrm{N}$, Isoda $\mathrm{H}$, Kanayama N, Terao $\mathrm{T}$. Vasospasms are characteristic in cases with eclampsia/preeclampsia and HELLP syndrome: proposal of an angiospastic syndrome of pregnancy. Semin Thromb Hemost. 2001;27:131-5.

26. Eiland E, Nzerue C, Faulkner M. Preeclampsia 2012. J Pregnancy. 2012;2012:586578.

27. Al-Jameil N, Aziz Khan F, Fareed Khan M, Tabassum H. A brief overview of preeclampsia. J Clin Med Res. 2014;6:1-7.

28. Hansen WF, Burnham SJ, Svendsen TO, Katz VL, Thorp JM Jr., Hansen AR. Transcranial Doppler findings of cerebral vasospasm in preeclampsia. J Matern Fetal Med. 1996;5:194-200.

29. Trommer BL, Homer D, Mikhael MA. Cerebral vasospasm and eclampsia. Stroke. 1988;19:326-9.

30. Belfort MA. The effect of magnesium sulphate on blood flow velocity in the maternal retina in mild pre-eclampsia: a preliminary colour flow Doppler study. Br J Obstet Gynaecol. 1992;99:641-5.

31. Wong CW, Wong TY, Cheng CY, Sabanayagam C. Kidney and eye diseases: common risk factors, etiological mechanisms, and pathways. Kidney Int. 2014;85:1290-302.

32. Moran P, Lindheimer MD, Davison JM. The renal response to preeclampsia. Semin Nephrol. 2004;24:588-95. 